\title{
PN2ARDUINO - A New Petri Net Software Tool For Control Of Discrete-event And Hybrid Systems Using Arduino Microcontrollers
}

\author{
Erik Kučera, Oto Haffner and Roman Leskovský \\ Faculty of Electrical Engineering and Information Technology \\ Slovak University of Technology in Bratislava \\ Bratislava, Slovakia \\ Email: erik.kucera@stuba.sk
}

\begin{abstract}
The main aim of proposed paper is the design of new software system for modelling and control of discrete-event and hybrid systems using Arduino and similar microcontrollers. In this article we propose a new tool. This new tool is based on Petri nets and it is called PN2ARDUINO. It offers a capability of communication with the microcontroller. Communication with the microcontroller is based on modified Firmata protocol so control algorithm can be implemented on all microcontrollers that support this type of protocol. The developed software tool was successfully verified for control of laboratory systems. It can also be used for education and also for research purposes as it offers a graphical way for designing control algorithm for hybrid and mainly discrete-event systems. Proposed tool can enrich education and practice in the field of cyber-physical systems.
\end{abstract}

\section{INTRODUCTION}

Development of various systems is a complex discipline that includes many activities, e.g. system design, a specification of required properties, implementation, testing and further development of the system. As these operations are challenging and important for the final product, it is appropriate and necessary to create a model of the system. Development of control methods of discrete-event and hybrid systems belongs to the modern trends in automation and mechatronics. Hybrid system is a combination of continuous and discrete event systems. Control of such systems brings new challenges because it is necessary to join control methods of discrete event systems (where formalism of Petri nets can be helpful) and classic control methods of continuous systems. With good methodology and software module, these approaches can be synergistically combined. This will give us an appropriate and unique control system that allows harmonizing discrete event control methods with the methods of control of continuous systems (e.g. PID algorithms). Effective cooperation of these approaches allows to control hybrid system. This method would be useful in systems where it is necessary to use different control algorithms (for example PID controllers with different parameters) according to the state of the system. The concept of Petri nets is capable of covering a management of these control rules in a very efficient, robust and wellarranged (graphical) way. This paper is aimed to present new
Petri Net tools for modelling and control of discrete-event and hybrid systems. Case studies for control of laboratory fire alarm system and DC motor are also presented.

In [1] author developed an interesting software tool that supports hybrid Petri nets named Visual Object Net++. There a lot of papers (mainly from Romanian author [2]) that describes capabilities of Visual Object Net++. This tool is not opensource and it is not further developed.

As an interesting way of research, a Modelica language and open-source tool OpenModelica appeared. There is a library that supports modelling by Petri nets in this tool. One of the advantages of OpenModelica is that PN model can be connected with other components of Modelica. The first Petri net toolbox was introduced in [3]. An extension of this toolbox was described in [4]. The greater addition to the toolbox was made by the German author who enriches it by a support of extended hybrid Petri nets for modelling of processes in biological organisms [5] and [6]. This tool was developed primarily for commercial tool Dymola and not for OpenModelica, so applicability in scientific research and extensibility is limited. During 2015 the team that developed PNlib published modified version of PNlib that partially worked in OpenModelica. Unfortunately, it was not possible to use OpenModelica for control purposes using microcontrollers because of lack of COM port communication support.

According to the survey made during the described research project, it was realized that it is necessary to develop the own solution for control of discrete and hybrid systems using Petri nets based on microcontrollers as there is a lack of tools that support control of real systems using Petri net formalism.

\section{Description of Developed SW Tool PN2ARDUINO}

As it was realized that there is no complex SW solution to support control of discrete event and hybrid system by microcontrollers using High-level Petri nets, it was necessary to develop it. As a basis for such software, PNEditor [7] was chosen. This tool is open-source. The developed extension of this tool is named PN2ARDUINO and is fully tested in [8] and [9]. The main topic of this paper is an introduction to this 


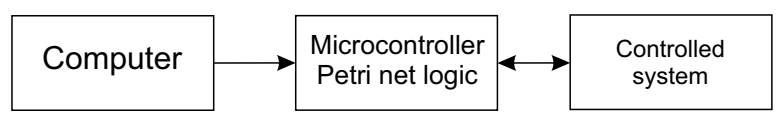

Fig. 1. Simple scheme of proposed solution - Petri net's logic in microcontroller

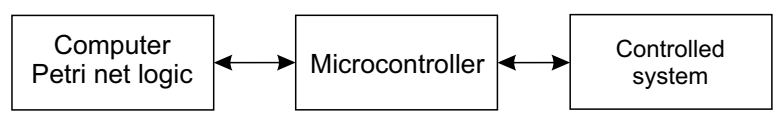

Fig. 2. Simple scheme of proposed solution - Petri net's logic in PC

developed software that can be used for control of discrete event and hybrid systems and its verification on laboratory discrete-event and hybrid system.

There are more concepts of control using Petri nets. Petri net as a control logic is necessary to connect with the controlled system (e.g. using microcontroller). One of the main aspects of the control system design is the question whether the Petri net's logic should be stored in the microcontroller or into the PC (which can communicate with microcontroller). Both approaches have their advantages and disadvantages.

If the Petri net's logic is stored in the microcontroller, the main advantage is the independence of control unit from the software application (program on PC). The Petri net logic is modelled using PC, and then the Petri net is translated into program code which is loaded into the microcontroller. Then PC and microcontroller can be disconnected. The advantage is also the capability of control in real time. Disadvantages are limited computational and memory resources of the microcontroller. Following disadvantage is the need of repeating compiling and uploading the program into the microcontroller (mainly during development phase). The proposed solution is shown in Fig. 1.

When the Petri net's control logic is stored in specialized SW application on PC, this solution gives an opportunity to control the system directly from it. In the microcontroller, only the program with communication protocol is stored. This communication protocol (in our case it is Firmata [10]) is used for communication between PC and microcontroller. This solution eliminates the necessity of recompiling and reuploading the program during development. The next advantage is the elimination of restrictions on computing and storage resources because PC has (in comparison with microcontroller) almost unlimited resources. One of the disadvantages is that the control system cannot react in real time. The proposed solution is shown in Fig. 2.

In Table I, these differences are specified.

New software module PN2ARDUINO was based on the second approach. The Petri net runs on the personal computer. For communication between SW application and microcontroller, the protocol Firmata [10] was used. Firmata is a protocol that is designed for communication between microcontroller and computer (or mobile device like a smartphone, tablet, etc.). This protocol can be implemented in firmware of various

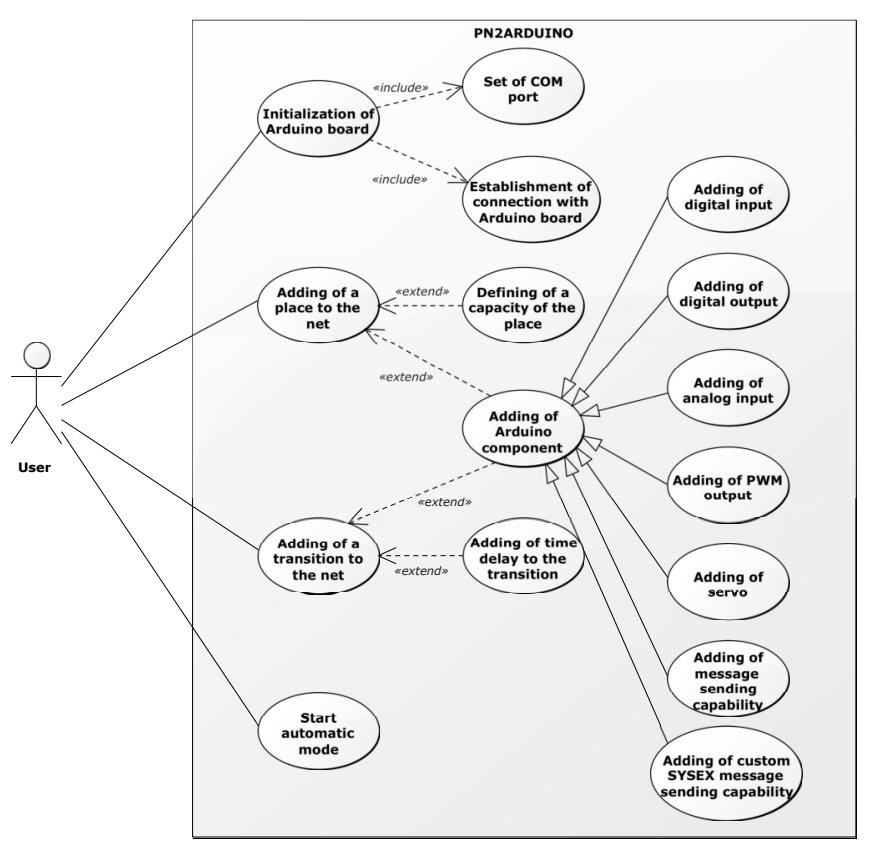

Fig. 3. PN2ARDUINO - Use-case diagram

microcontrollers. Mostly Arduino-family microcontrollers are used. On PC the client library is needed. These libraries are available for many languages like Java, Python, .NET, PHP, etc. Firmata protocol is based on MIDI messages [11].

On the Arduino side, Standard Firmata 2.3.2 version is used. The client application on PC is based on Firmata4j 2.3.3 library which is programmed in Java. The advantage of using Firmata is that another microcontroller compatible with Firmata can be used.

PN2ARDUINO extends PNEditor with many features. For Petri nets modelling, there is a capability of adding time delay to transitions and capacity for places. Also, automatic mode of firing transition was added for automatic system control purposes as only manual mode was present in PNEditor.

PN2ARDUINO brings a new communication module to PNEditor. This module communicates with the compatible microcontroller. This module consists of two parts. The first one provides the creation of connection with the microcontroller, so it sets COM port where the microcontroller is connected. The second part provides the implementation of a capability of adding Arduino components to Petri net's places and transitions. These types of Arduino components are supported: digital input and output, analog input, servo control, PWM output, message sending, custom SYSEX message [10] sending.

In Fig. 3, the use-case diagram of developed SW tool can be seen.

As it was stated, transitions and places can be associated with Arduino components. Digital and analog inputs serve as enabling conditions for transitions in Petri net. Digital and PWM outputs and messages serve as the executors of the respective actions. 
TABLE I

COMPARISON OF TWO CONCEPTS OF SYSTEM CONTROL USING PETRI NETS

\begin{tabular}{|c|c|}
\hline Petri net logic in PC & Petri net logic in microcontroller \\
\hline limited capability of real-time control & real-time control \\
\hline much more computation and memory resources available & limited computation and memory resources \\
\hline code in microcontroller does not need recompiling & during development repeated compiling is needed \\
\hline PC must be still online & independence of control unit \\
\hline
\end{tabular}

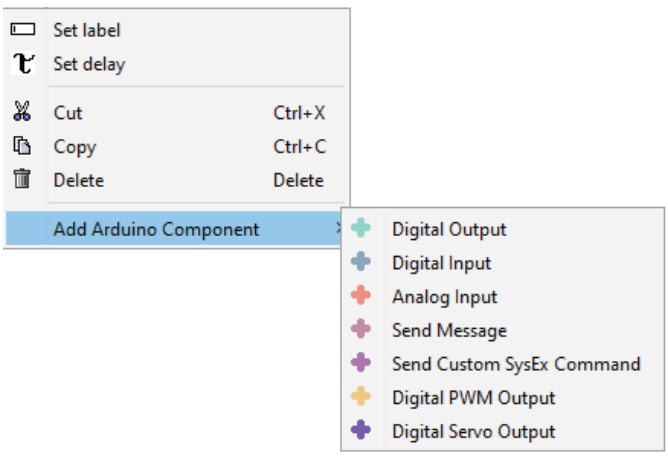

Fig. 4. PN2ARDUINO - Adding of Arduino component

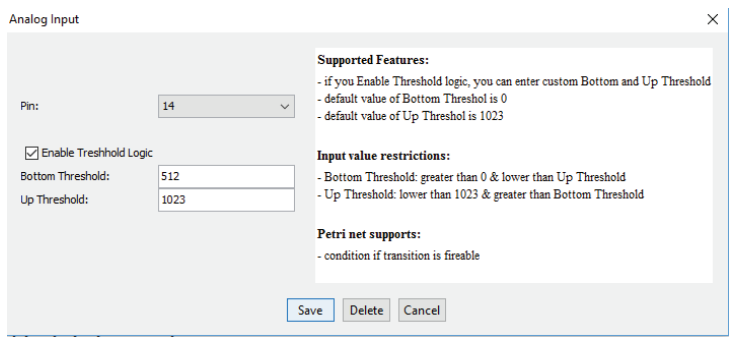

Fig. 5. PN2ARDUINO - Analog input

The interesting functionality is a capability of sending custom SYSEX messages. The user must enter SYSEX command $(0 \times 00-0 \times 0 F)$ and optionally also the content of the message. The message is sent when the token comes to the place or when the transition is fired. For example, SYSEX messages are used in the proposed example of hybrid control in the last section of the paper. Here, the SYSEX message notifies the microcontroller that a different PID algorithm should be used for system control. Then PID algorithm is switched, and the controlled system remains stable.

A main window of PN2ARDUINO consists of a quick menu, main menu, canvas for Petri net modelling and log console. PN2ARDUINO supports two modes - design mode and control mode. Control mode is manual and automatic.

Firstly, it is necessary to initialize communication with Arduino (Setup board in the menu). Then it is possible to add Arduino component to the place or the transition (Fig. 4). The example of analog input can be seen in Fig. 5.

Time politics are also supported. To the transitions, it is possible to add time delay which can be deterministic or stochastic.

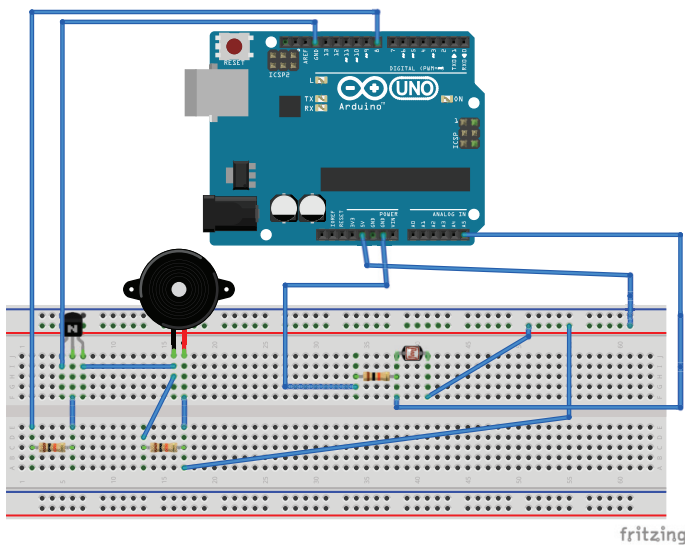

Fig. 6. The scheme of laboratory model of fire alarm

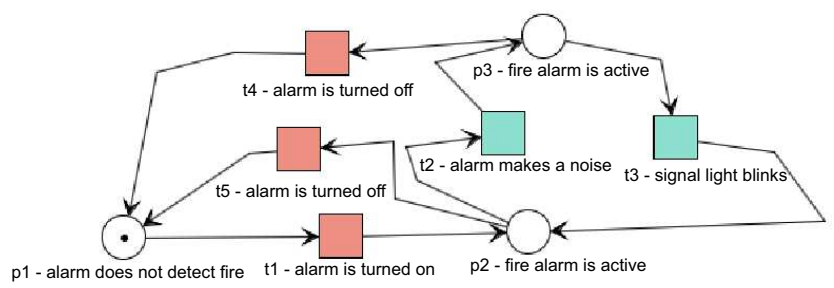

Fig. 7. PN for fire alarm (initial marking)

\section{CASE STUdy: CONTROL OF LABORATORY DISCRETE-EVENT SYSTEM}

For verification of proposed software tool and method of discrete-event systems control it was necessary to design an education laboratory model of such system. A fire alarm model was built. The scheme can be seen in Fig. 6 .

This model consists of an active buzzer, photo-resistor, three resistors and NPN transistor. NPN transistor is mandatory for active buzzer connection. The LED of Arduino in pin 13 is also used. Photo-resistor was used instead of the smoke sensor because of the less complicated feasibility of experiment.

Then the behaviour of the system must be defined. When the photoresistor detects an excessive lighting (it was experimentally determined as input value greater than 799 on the analog pin of Arduino Uno which resolution is from 0 to 1023) the intermittent tone of the buzzer is turned on. This tone alternates with LED lighting. When the value on the analog pin lowers below 800 , these sound and light effects stop. This is repeated cyclically. 


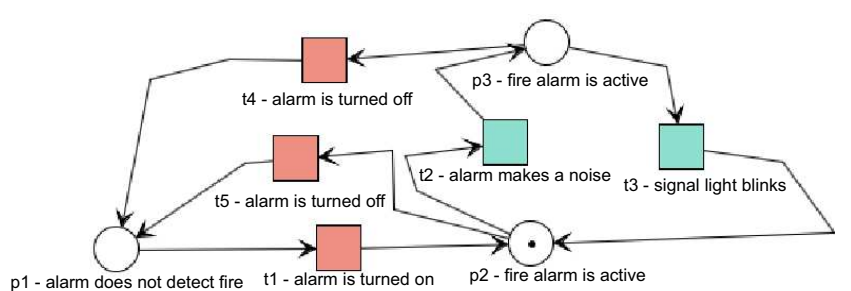

Fig. 8. PN for fire alarm $\left(t_{1}\right.$ is fired $)$

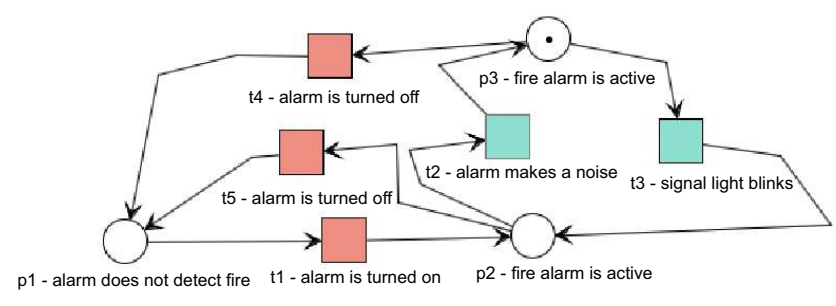

Fig. 9. PN for fire alarm ( $t_{2}$ is fired)

Initial marking of modelled timed Petri Net interpreted for control (or sometimes called as interpreted timed Petri net) in PN2ARDUINO is shown in Fig. 7.

Places of Petri net (Fig. 7 - Fig.9) corresponds with these states:

- $p_{1}$ - alarm does not detect fire

- $p_{2}$ and $p_{3}$ - alarm is active (fire was detected)

Transitions of Petri net (Fig. 7 - Fig.9) corresponds with these actions/events:

- $t_{1}$ - alarm is turned on

- $t_{2}$ - alarm makes a noise

- $t_{3}$ - signal light blinks

- $t_{4}$ and $t_{5}$ - alarm is turned off

The token is in place $p_{1}$ which corresponds with the state when the fire alarm is not activated because the photo-resistor does not detect light intensity threshold.

At the time when the value greater than 799 is detected on the analog pin of Arduino - the transition $t_{1}$ is fired. This transition is associated with Arduino component Analog Input where a range of input values is set. This range determines when the transition is enabled.

Now the token is in the place $p_{2}$ (Fig. 8). Transition $t_{2}$ is associated with Arduino component Digital Output (in this case pin 8) where the buzzer is connected. This transition has also associated the function of time delay - 2 seconds. That means that transition firing (and sound effect of buzzer) lasts for 2 seconds.

Now the token is in the place $p_{3}$ (Fig. 9). Transition $t_{3}$ is associated with Arduino component Digital Output (in this case pin 13) where the build-in LED is connected. Time delay is set to 1 second. LED diode turns on for 1 second.

This process is repeated cyclically, and it is stopped when the value on the analog pin is lowered under the value 800 . Then the transition $t_{4}$ or $t_{5}$ is fired and token moves to the place $p_{1}$ when fire alarm does not detect the fire.

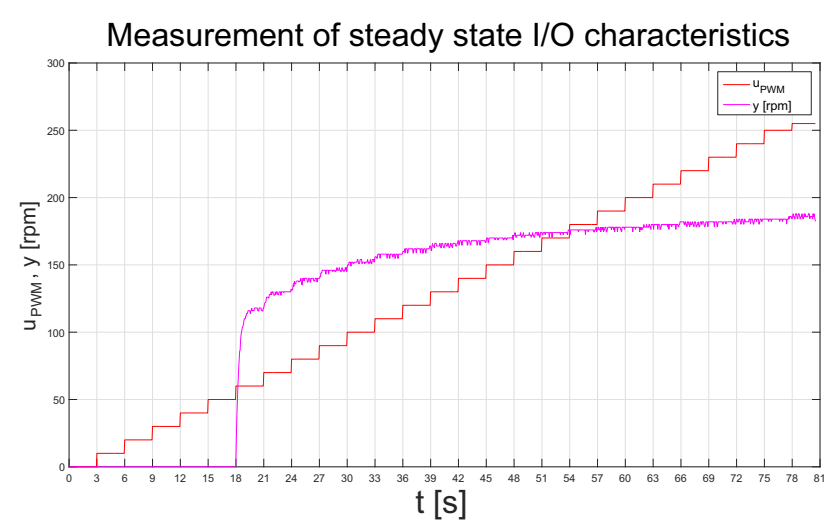

Fig. 10. Measurement of steady state I/O characteristics of DC motor

We can conclude that the ability of discrete-event control with PN2ARDUINO was successfully verified a generalized for other applications.

\section{CASE STUDY: CONTROL OF LABORATORY HYBRID SYSTEM}

For verification of proposed software tool for hybrid systems control, it was necessary to design a laboratory model of such system. A DC motor with encoder was chosen. The encoder is used for feedback in the system because it is used for speed measurement. The actual speed of the DC is in is measured process value.

DC motor was connected to Arduino Uno using the motor shield module. Arduino motor shield is based on dual full bridge driver L298. Using the motor shield, it is possible to independently control speed and motion direction of DC motor. The encoder in this motor is of incremental type. For speed measurement, it is necessary to use hardware interruptions functionality of Arduino Uno.

The speed of the motor is set by pin described as "PWM A". When the input is set to "PWM $=255$ " the Arduino program shows $186 \mathrm{rpm}$ which approximately corresponds with parameters stated by the manufacturer.

The next step was a measurement of steady state I/O characteristics. The input is a voltage supplied to the motor. These inputs are of size from $0 \mathrm{~V}$ to $5 \mathrm{~V}$ which corresponds with PWM signal from 0 to 255 (8-bit resolution). Sampling is 0.05 seconds. In Fig. 10 the process of measurement of steady state I/O characteristics is shown. The signal was filtered by 1-D median filter of 2 nd order. Red line is input to the system (voltage or PWM). Output (rpm) is shown by magenta line. Steady state I/O characteristics is in Fig 11.

In the process of working points choosing it was necessary to choose points which meet the certain condition. This condition is that behaviour of the system must be close to the linear behaviour around these points. From I/O characteristics two values of input $\left(u_{P_{1}}\right.$ and $\left.u_{P_{2}}\right)$ and output $\left(y_{P_{1}}\right.$ and $\left.y_{P_{2}}\right)$ were chosen (these values will be our working points):

$$
u_{P_{1}}=80 \rightarrow y_{P_{1}}=140 \mathrm{rpm}
$$




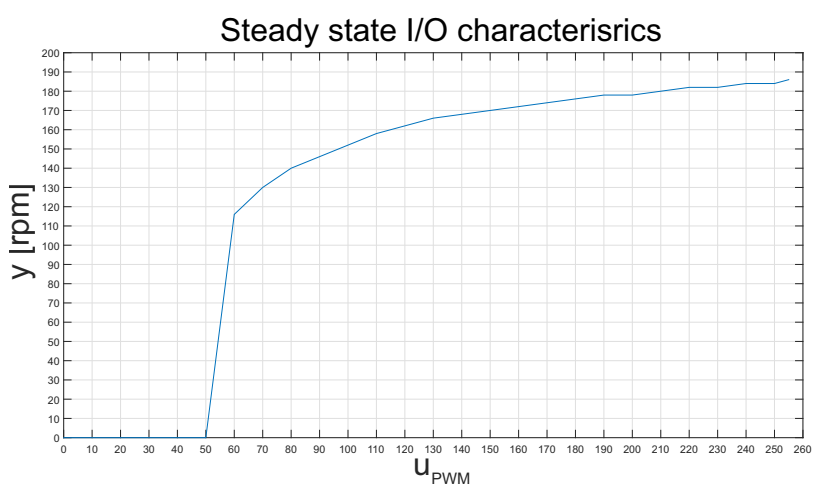

Fig. 11. Steady state I/O characteristics of DC motor

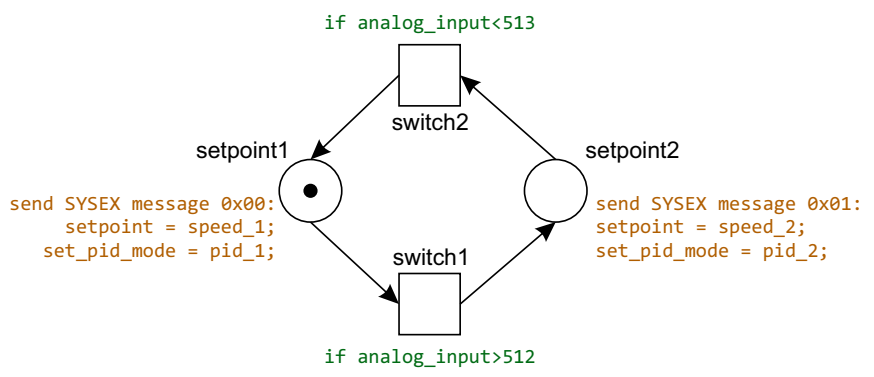

Fig. 12. Control scheme for hybrid system using PN2ARDUINO

$$
u_{P_{2}}=170 \rightarrow y_{P_{2}}=174 r p m
$$

From solution analysis, it is obvious that for each working point it is necessary to use a different controller. One of the solutions is an option to switch between multiple controllers according to the working point - speed (rpm) of DC motor. It is possible to use developed software module PN2ARDUINO. It is possible to switch between controllers and setpoints using SYSEX messages. Arduino and other microcontrollers that support Firmata protocol can be used. Development and verification of this software module are one of the most interesting results of presented research.

For illustration see the scheme in Fig. 12. It is an example for a demonstration of proposed control method. Assume the mentioned DC motor. We require to operate it in 2 modes (working points or rpm). For effective settlement of speed value to the setpoint, controllers with different parameters are needed (different controller for each mode). We switch between rpm using potentiometer connected to the analog input of microcontroller Arduino Uno. The switching between controllers is provided by transitions of Petri net named switch 1 and switch 2 according to the input value from potentiometer. Input from the analog pin in Arduino is represented by value between 0 and 1023 . As the threshold, a half value was used (512). In the moment when the token in Petri net is moved to the place named setpoint1 or setpoint 2 , a SYSEX message is sent. This message ensures the execution of user defined program code on the Arduino side. In this case, the control algorithm is executed. An algorithm (PID controller) for continuous control is independent of Firmata messaging, so it provides real-time control.

The case study of hybrid systems control proposed a basic example. Researchers in the field of hybrid control design can use it for different and more complicated scenarios.

\section{CONCLUSION}

The paper presents the new software tool named PN2ARDUINO which extends PNEditor with the capability of communication with microcontrollers that supports Firmata protocol. Then it is possible to control discrete-event and hybrid systems using timed interpreted Petri nets with developed software tool. This tool uses the control paradigm when the microcontroller has implemented only the communication protocol. Petri net's control logic is stored in the computer which communicates with the microcontroller and sends control orders. The next research will focus on the concept of control with Petri nets where control logic will be directly implemented on the microcontroller.

\section{ACKNOWLEDGMENT}

This work has been supported by the Cultural and Educational Grant Agency of the Ministry of Education, Science, Research and Sport of the Slovak Republic, KEGA 030STU4/2017 and KEGA 038STU-4/2018, by the Scientific Grant Agency of the Ministry of Education, Science, Research and Sport of the Slovak Republic under the grant VEGA $1 / 0733 / 16$, and by the Young researchers support program, project No. 1328 - KVPRI (Quality Control of Production Processes with Augmented Reality in Industry 4.0) and project No. 1327 - VTOVI (Virtual Training of Production Operators for Industry 4.0).

\section{REFERENCES}

[1] H. Matsuno, A. Doi, R. Drath, and S. Miyano, "Genomic object net: object oriented representation of biological systems," Genome Informatics Series, pp. 229-230, 2000.

[2] M. A. Drighiciu and G. Manolea, "Application des reseaux de petri hybrides a l'etude des systemes de production a haute cadence," 2010.

[3] P. J. Mostermany, M. Ottery, and H. Elmqvistz, "Modeling petri nets as local constraint equations for hybrid systems using modelica," retrieved online at http://citeseer. ist. psu. edu/359408. html, 1998.

[4] S. Fabricius and E. Badreddin, "Modelica library for hybrid simulation of mass flow in process plants," in Proceedings of the 2nd International Modelica Conference', Oberpfaffenhofen, Germany. Citeseer, 2002, pp. 225-234.

[5] S. Proß, B. Bachmann, and A. Stadtholz, "A petri net library for modeling hybrid systems in openmodelica," in submitted (Modelica Conference 2009), 2009.

[6] S. Proß and B. Bachmann, "Pnlib-an advanced petri net library for hybrid process modeling," in Modelica Conference, 2012.

[7] M. Riesz, M. Seckár, and G. Juhás, "Petriflow: A petri net based framework for modelling and control of workflow processes." in ACSD/Petri Nets Workshops. Citeseer, 2010, pp. 191-205.

[8] A. Cesekova, "Control of laboratory discrete event systems (in slovak)," Master's thesis, Slovak University of Technology in Bratislava, 2016.

[9] E. Kucera, "Modelling and control of hybrid systems using highlevel petri nets (in slovak)," Ph.D. dissertation, Slovak University of Technology in Bratislava, 2016.

[10] H.-C. Steiner, "Firmata: Towards making microcontrollers act like extensions of the computer." in NIME, 2009, pp. 125-130.

[11] M. Association. (2016) Summary of midi messages. [Online]. Available: https://www.midi.org/specifications/item/ table-1-summary-of-midi-message 